\title{
PARTICLE-IN-CELL CODE LIBRARY FOR NUMERICAL SIMULATION OF THE ECR SOURCE PLASMA
}

\author{
V. Alexandrov, A. Filippov, N. Kazarinov, R. Komissarov, V. Mironov, V. Preisendorf, \\ V. Shevtsov, G. Shirkov, E. Shirkova, O. Strekalovsky, N. Tokareva, and A. Tuzikov, Joint Institute for Nuclear \\ Research, Dubna, Moscow region, 141980, Russia \\ A. Anisimov, E. Donskoi, V. Fomin, A. Golubev, S. Grushin, V. Povyshev, A. Sadovoi, E. Vasina, V. Vatulin, and R. \\ Veselov, Russian Federal Nuclear Center - Scientific Research Institute for Experimental Physics, (VNIIEF), \\ Sarov, Nijni-Novgorod Region, 607190 Russia \\ V. Shevelko, Lebedev Physical Institute of Russian Academy of Science, Moscow, Russia \\ T. Nakagawa, and Y. Yano, The Institute of Physical and Chemical Research (RIKEN), 2-1 Hirosawa, Wako, Saitama \\ 351-01, Japan
}

\section{Abstract}

The code library is aimed for the three-dimensional simulation of the electron cyclotron resonance (ECR) plasma and ion production in an ECR ion source. The particle-in-cell method allows studying the detailed characteristics of plasma, taking into account distribution functions of particles, real self and external fields, particle-particle interactions, and others.

\section{INTRODUCTION}

The practical realization of the project "Numerical simulation and optimization of ion accumulation and production in multicharged ion sources" was started in June 1999. This project is to be funded by International Science and Technology Center (ISTC) and is carried out in the frame of RIKEN Project of Radioactive Isotope (RI) Beam Factory [1]. The factory consists of a cascade of ion accelerators and accumulator rings and is aimed at providing RI beams over the whole atomic mass range with the high level of intensity in a wide range up to several hundreds $\mathrm{MeV} /$ nucl. A first link of the accelerator chain will be a new superconducting electron cyclotron resonance (ECR) ion source. The physical substantiation of ISTC project was prepared and published in RIKEN in 1997 [2].

The project goal is a creation of new generation model and codes for the numerical simulation based on the particle-in-cell method. This is one of finite particle methods for the simulation of multicomponent ECR plasma and electron-ion beams. These methods are the most powerful methods for the numerical simulation of motion of continuous medium, gas and plasma dynamics, and dynamics of charged particle beams. The finite particle methods allow studying detailed characteristics of continuous medium: distribution functions of particles (spatial, velocity and energy distributions), real self and external fields, particle-particle interactions and many other effects. This technique allows providing very precise numerical simulations of the ECR plasma and optimizations of ECR ion sources in this way.

The finite particle methods are Euler or Lagrange methods for mathematical describing of a continuous medium [2]. In the Euler method for a continuous medium the physical system is considered at definite mesh points. The combination of both methods is the most effective for simulations in the physics of plasma or charged particle beams. In the particle-in-cells methods the motion of particles is described in Lagrange variables and, simultaneously, the particle densities, currents and fields are determined in a stationary mesh or in Euler variables.

The aim of the project is creation of three-dimensional (3D) code library for the plasma dynamics and ion production simulation in ECR source. The code must simulate dynamics of all plasma components, radio frequency (rf) electron heating, ion production, accumulation and extraction, all external and self fields, interaction of plasma particles with source walls and elements of construction and so on.

The project includes preliminary investigations and development of physical and mathematical models of processes in multicomponent plasma. These results have been published in advance as preprints of RIKEN and JINR [2-5]. A summary of those results as well as results of the first testing of computer code library are presented here.

\section{PRELIMINARY INVESTIGATIONS}

\subsection{Verification of cross-sections of electron impact ionization [4]}

To increase the accuracy of calculated cross sections of electron impact ionization and sufficiently improve the widely used Lotz formula [2] in comparison with experimental data it was proposed to describe total cross sections with a new parametrical formula:

$$
\sigma(E)=10^{-13}\left(\frac{1}{I E}\right)^{2}\left[\sum_{i=1}^{6} A_{i}\left(1-\frac{I}{E}\right)^{i}+B \cdot \ln \left(\frac{E}{I}\right)\right]
$$

where $E$ is electron impact energy $(\mathrm{eV}), I$ is ionization potential of valence electron, and Bethe constant $B$ determines the cross section value for high energy of the incident electron. The number of parameters $A_{i}$ was selected in order to describe total ionization cross sections for practically all neutral atoms between $\mathrm{He}$ and $\mathrm{U}$ and all their ions with a single formula. The parameters $A_{i}$ and $B$ are found applying the least-square root method to the known experimental and numerical data. 
Total ionization cross sections with an accuracy of about $10 \%$ in comparison with available experimental data were calculated on the basis of formula (1) for the neutral atoms and all their ions: $\mathrm{He}, \mathrm{N}, \mathrm{O}, \mathrm{Ar}, \mathrm{Kr}, \mathrm{Xe}, \mathrm{U}$ and others. The new improved formula (1) for precise fitting of ionization cross sections in wide range of electron impact energy has important meaning out of frames of this project and can be used in any atomic physics and plasma investigations instead of famous Lotz formula.

Atomic and ionic ionization potentials are required to calculate the cross-sections of atomic processes (ionization, charge exchange, recombination and etc.). The special library of data files was created for this purpose [5].

\subsection{Interaction of charged particles of plasma with walls and elements of construction}

The calculations of the secondary electron emission from the source walls were carried out using the code "Eliza" developed in VNIIEF (Sarov) [6]. The system of linear non-stationary Boltzmann equations is solved using the Monte Carlo method in this code.

The obtained results show [3] that total fast secondary electron yield is practically independent of initial primary electron energy in $\mathrm{keV}$ region, but strongly depends on the incidence angle. Average energy of secondary electrons is practically independent of the oxide layer thickness, but, naturally, depends on the primary electron energy, and is of $60 \div 80 \%$ of primary electron energy. Significant influence of the wall oxide layer is observed only for primary electron energies below $2 \mathrm{keV}$ because of comparable stopping range of produced secondary electrons and the oxide layer thickness.

\section{DEVELOPMENT OF PHYSICAL AND MATHEMATICAL MODELS}

\subsection{Calculation of external electromagnetic fields}

Electrical and magnetic fields are created by a system of coils, permanent magnets and special electrodes in the ECRIS. These fields can be calculated in analytical or numerical ways or can be set as an experimental data table. The following models and procedures have been chosen for using for simulations [3]: analytical equations for field calculations of typical elements: solenoids, quadrupole or multipole lenses, horizontal and vertical bending magnets (both of sector type and with the pole face rotation $\alpha$ and bending radius $\rho$ ), accelerate cavities and drift spaces; POISSON/SUPERFISH codes [7] for field calculations of user defined sources: permanent magnets, solenoids with iron, "einzel" lenses, electrodes, etc; and data tables of experimental fields.

The quadratic interpolation is used to calculate fields at every point inside of cells.

\subsection{Plasma field calculations}

The plasma field determination is based on the solution of Poisson equations for the self and external fields [2].
Self field of particles is obtained from direct solution of Poisson's equation for electrostatic potential at every time step in the cylindrical system of coordinates with Dirichlet boundary condition at the conductive surface and with Neumann condition at the axis. The double Fourier transformation is used to solve the Poisson's equation. The procedure of quadratic interpolation is used to calculate the values of self-fields in the every point of every particle position.

\subsection{Dynamics of charged particles in plasma}

Particles of all masses and charge states are available in the consideration: ions of all charge states of all elements, different kinds of neutrals and electrons. Each finite particle represents the group of physical particles. Thus every finite particle species have masses and charges according to types of ions, neutrals or electrons.

Numbers of finite particles and cells of mesh determine the capacity, accuracy and resolution of simulation procedure. These values are limited usually by the power of computer for numerical simulation purpose.

\subsection{Simulation of atomic processes in plasma} $[8,9]$

Basic inelastic nuclear processes providing the change of a particle charge are ionization of neutral atoms and ions by electron impact; charge exchange of ions on neutral atoms; and ion recombination by low energy electrons.

The balance equations for ion densities are a consequence of the kinetic equations for ion distribution function and a ground of the algorithm to simulate inelastic processes in ECR plasma. These equations take into account all processes of ion production and loss and have a stochastic basement. It is quite evident to use a Monte-Carlo method of stochastic modeling to find a solution of the balance equations at each cell of mesh in simulations.

\subsection{Amounts of macro particles and cells for plasma description}

The complete amount of macro particles should be much higher than the amount of cells. Supposing that the amount of macro particles - ions of each charged state in an elementary cell is equal to $10-15$, the amount of various ions species does not exceed of 10-20, and taking into account that the plasma is electrically quasi neutral, we obtain that the macro particle amount must be in order of $(2-3) \times 10^{5}$ for the adequate plasma description. The relative error in definition of plasma field will not exceed the values of $1 / N^{I / 2}=1 /\left[(2-3) \times 10^{5}\right]^{1 / 2} \approx 2 \times 10^{-3}$, i.e. less than $1 \%$. In a three-dimensional case the amount of the finite particles must be increased by a factor of 16 at least and is not less of (3-5) $\times 10^{6}$.

\subsection{Integration method of motion equations}

Each finite particle has 6 coordinates in the $6 D$-phase space. It means that it has space coordinates $(x, y, z$ in Cartesian coordinates) and velocity or momentum 
coordinates. Equations of motion describe the changes in time of all of phase coordinates. The most economic numerical methods for integration of motion equations is a leap-frog method of the second order [3].
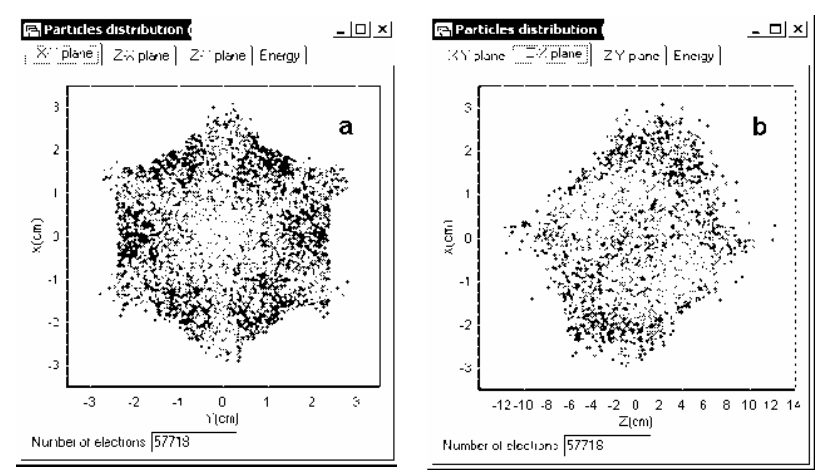

Fig.1 Distributions of electrons in $X Y$ (a) and $X Z$ (b) planes.

\section{FIRST TEST OF COMPUTER CODE LIBRARY}

The computer code library for ECR plasma simulation is created using the object-oriented programming. Output data of the programs are arrays of input coordinates and momentums of finite particles (neutrals and ions) taking into account the simulation of inelastic atomic collisions and transitions of particles if necessary.

The common WINDOWS interface of the library is aimed to set parameters and initial conditions, to start the program of the ECR ion source simulation and to control the calculation process. The main menu of the program includes the following items: General, Initial conditions, Calculations, View results.
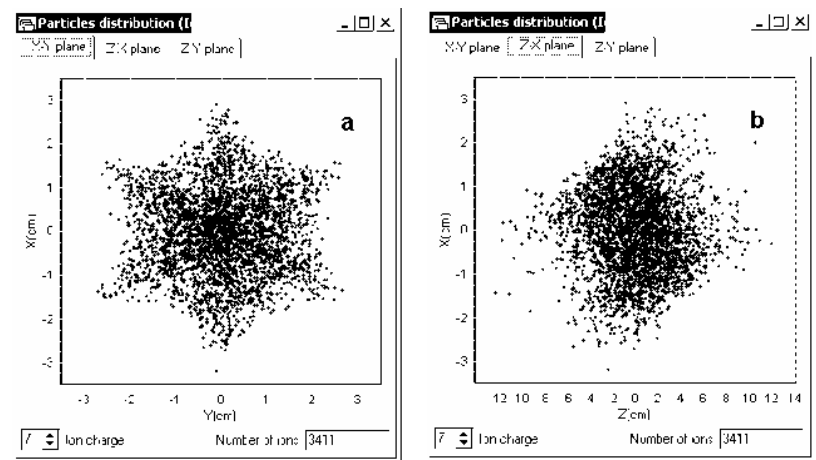

Fig.2. Distributions of $\mathrm{N}^{7+}$ ions in $X Y(\mathrm{a})$ and $X Z$ (b) planes

The first tests of computer code library are presented in Figs 1-3. These figures show results of a model simulation of nitrogen ion production and accumulation during $3.5 \mathrm{~ms}$ in the $18 \mathrm{GHz}$ ECR source with average electron density of $2 \times 10^{12} \mathrm{~cm}^{-3}$, longitudinal magnetic field with trap longitudinal configuration $\left(B_{z}=0.47 \mathrm{~T}, \mathrm{Z}\right.$ $=0$ and $B_{z}=1.5 \mathrm{~T}, Z= \pm 12 \mathrm{~cm}$ ) and sextupole azimuthal variation. These figures have been taken from computer screen and can give some impressions of the user interface: numbers of macro particles in considerations, items of user menu, charge states of ions and etc.

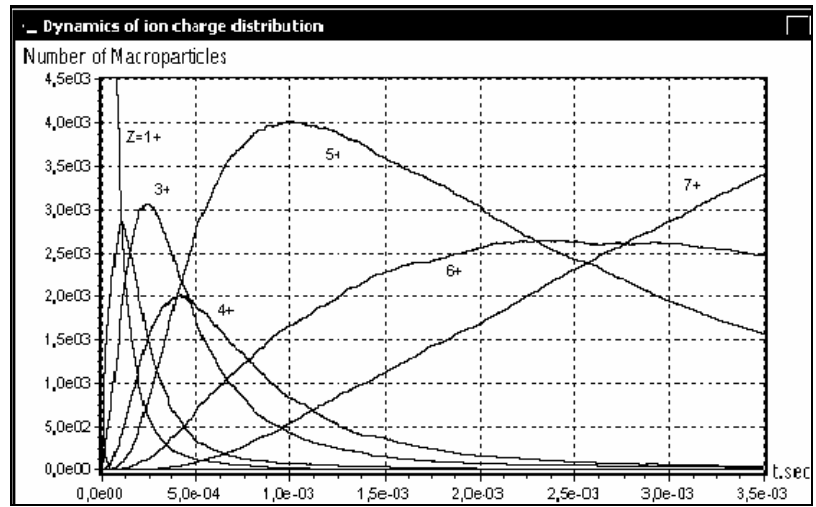

Fig.3. Ionization dynamics of nitrogen ions.

Figure 1 shows spatial distributions (a for $X Y$ plane and b for $X Z$ plane) of two components of electrons. The light color of macro particles corresponds to a low energy electron component and the dark color corresponds to a high energy one in saturation as the result of rf electron heating. The image of this distribution is created by almost 60000 macro particles of cold and hot electron components. Figure 2 shows spatial distributions (a for $X Y$ plane and $\mathbf{b}$ for $X Z$ plane) nitrogen ions of the seventh charged state. The shapes of spatial distributions of electrons and ions are extremely correlated with longitudinal and azimuthal distributions of external magnetic field in the source. Evidently the process of ion creation depends on spatial distribution of plasma components. A development of ion ionization and accumulation is presented in Figure 3. These results are the first experience only to test and to demonstrate the code library potentialities.

\section{REFERENCES}

[1] Y.Yano, A.Goto, T.Katayama In: Collected papers on RIKEN RI Beam Factory Project, December 1998,RIKEN.

[2] G.Shirkov, Y.Batygin, Y.Yano, preprint RIKEN-AFAC-5, 1997, 36pp.

[3] G. Shirkov et al," Preprint RIKEN, 2000, RIKEN-AFAC-24, 58 p.

[4] V.M. Povyshev, et al, preprint JINR, E9-2001-148, JINR, Dubna, 2001.

[5] C.Muhle, E.Shirkova, G.Shirkov. JINR Communication P9-93-307, Dubna, 1993, 5p.

[6] Donskoi E.N, Voprosy Atomnoi Nauki I Tekhniki. Ser. Mathematical Modeling of Physical Processes. 1993. Issue. 1, pp. 3-6.

[7] M.T.Menzel, H.K.Stokes, POISSON/SUPERFISH Group of Codes, LA-UR-87-115, Los Alamos, New Mexico, 1987.

[8] G.Shirkov, G.Zschornack. Electron Impact Ion Sources for Highly Charged Ions. GWV Verlagsgesellschaft, Vieweg \& Sohn, Wiesbaden 1996, $310 \mathrm{pp}$.

[9] G.Shirkov, Plasma Sources Sci. Technol. 2 (1993), p. 250 . 